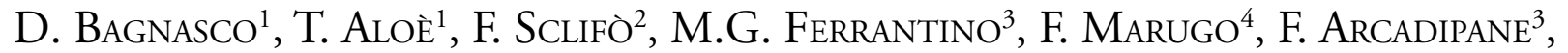
A. Manfredi ${ }^{1}$, G.W. Canonica ${ }^{5}$, G. Passalacqua ${ }^{1}$

\title{
When to stop biologicals. Severe asthma exacerbation after mepolizumab discontinuation
}

\author{
${ }^{1}$ Allergy and Respiratory Diseases, IRCCS Policlinico San Martino, University of Genoa, Genoa, Italy \\ ${ }^{2}$ Emergency Department, Ospedale Policlinico San Martino, Genoa, Italy \\ ${ }^{3}$ Respiratory Diseases, Azienda Ospedaliera Nazionale SS. Antonio e Biagio e C. Arrigo, Alessandria, Italy \\ ${ }^{4}$ Respiratory Diseases, Ospedale La Colletta, Arenzano, Italy \\ ${ }^{5}$ Personalized Medicine Clinic Asthma and Allergy, Humanitas Clinical and Research Center, Department of Biomedical \\ Sciences, Humanitas University, Rozzano, Milan, Italy
}

\begin{abstract}
KEYWORD
mepolizumab; real-life; discontinuation; severe asthma; monoclonal antibodies
\end{abstract}

\section{Corresponding Author}

Diego Bagnasco

Allergy and Respiratory diseases

IRCCS Policlinico San Martino

Largo Rosanna Benzi 10

16132, Genoa Italy

Phone: +390105555805

E-mail: diego.bagnasco@dimi.unige.it

Doi

10.23822/EurAnnACI.1764-1489.88

\section{To the Editor}

Severe asthma affects $5 \%$ to $10 \%$ of all asthmatic patients (1). To be defined as severe an asthmatic, patient must be treated with the best standard therapies at maximal doses, without achieving a full control, and therefore requiring also oral corticosteroids (OCS) (2). In Western Countries, severe asthma is responsible for at least $50 \%$ of the socio-economic burden, including both direct and indirect costs (3). The development of monoclonal antibodies (mAbs) allowed to expand the therapeutic approach in patients affected by this disease, especially in reducing the need for OCS and the exacerbation rate. Certainly, the most studied immunological aspect is that linked to the "type 2" inflammation, which involves eosinophils and other factors through the action of several cytokines, including interleukin (IL) 5 (4). Severe asthma also involves unrecognized or underestimated social costs, for instance due to the effects of systemic corticosteroids or work absenteeism (5). The novel biological drugs for severe asthma are able to reduce the direct and indirect costs, while maintaining a satisfactory control of disease. Mepolizumab (MEP), a humanized $\mathrm{mAb}$ against IL-5 was recently tested and commercialized for severe asthmatic patients $(6,7)$.

We describe herein the clinical case of a patient with severe hypereosinophilic asthma, successfully treated with MEP, first during the MENSA (6) study, then with its open-label extension and commercial availability, who had a sudden episode of asthma exacerbation after MEP withdrawal after more than 3 
years of therapy. Written informed consent was obtained from the patient for publication of this case report.

A never-smoker 64-year-old Caucasian male was followed-up by our clinic since about 10 years due to severe uncontrolled asthma. Asthma appeared around the age of 40, without any previous evidence of respiratory symptoms. Of note, between 40 and 60 years of age, the patient underwent 5 surgical interventions for nasal polyposis. Besides, an episode of isolated hypereosinophilia without symptoms was recorded. During clinical and historical examination, we found gastro-oesophageal reflux and a positive skin prick test to Parietaria. There was no evidence of occupational exposure to pulmonary pathogens. In the 2 years before enrolling him in MENSA study respiratory symptoms became increasingly severe, with a significant limitation to daily activity, despite the maximal therapy (fluticasone / salmeterol 50/500 mcg twice daily, theophylline 300 $\mathrm{mg} / \mathrm{die}$, proton-pump inhibitors and nasal steroids). To exclude that symptoms are related to a poor adherence to the therapy, we carefully checked it.

In the two years before enrolling him in MENSA, a mean of 4 asthma exacerbations per year were ascertained, with multiple hospital admissions. Finally, at the end of 2012 the patient was enrolled in the MEA 115588 trial (MENSA) (6), and treated with MEP. The baseline characteristic before MEP administration, was an increased number of blood eosinophils (770 cell/ $\mu \mathrm{l})$, a normal lung function, a mild hypertension and a normal saturation. At the beginning of 2013 the patient was randomized and included in the study. After the first month of therapy a clinical improvement could be observed, with an improvement in $\mathrm{FEV}_{1}$ (from $2.45 \mathrm{~L}$ to $2.87 \mathrm{~L}$ ). Afterwards the patient continued, with subjective and clinical benefit, with no more exac- erbations or systemic steroids need. At the end of the study, he received regularly MEP for compassionate use then, after marketing, he continued for other 8 months without symptoms or drug related adverse events.

After 4 years and 11 months of therapy, at the end of November 2017, with a full control of asthma symptoms, no exacerbations, no OCS therapy and normal lung function, we attempted MEP discontinuation. After about 2 months of control we could assist to a progressive deterioration: worsening of respiratory symptoms with dyspnoea even at rest, wheezing, cough, and nocturnal awakenings due to shortness of breath and a normal haemoglobin saturation. His blood eosinophils level increased to 820 cell/ $\mu \mathrm{l}$ and lung function test showed a progressive impairment. A home-based treatment with OCS (50 mg/day Prednisone), aerosolized steroids and bronchodilators was started, without a clinical improvement. The patient was hospitalized and treated with intravenous steroid and aminophylline. During the hospitalization, a bacterial superinfection occurred, for which an antibiotic therapy was set. A progressive objective and subjective improvement of symptoms was seen, with a reduction of blood eosinophils, and $500 \mathrm{~mL} \mathrm{FEV}$ improvement. After this, the MEP therapy was re-started during hospitalization and, in his clinical stability, patient was discharged. Currently, the patient is on regular MEP treatment, and remains well controlled, without exacerbations or OCS treatment needed (Table I).

All clinical trials with MEP, after appropriately selecting patients (serum eosinophils > 300/ $\mu$ l), confirmed the positive results in reducing exacerbations (6,7), in sparing OCS (8), in FEV1 improvement and in quality of life (6-8). In the current trials, a reassuring safety profile was also demonstrated with a small number of adverse events, similar to the placebo groups (9).

Table I - Main data at baseline, after MEP therapy (MEA 115588, compassionate use and marketing), during and after exacerbation.

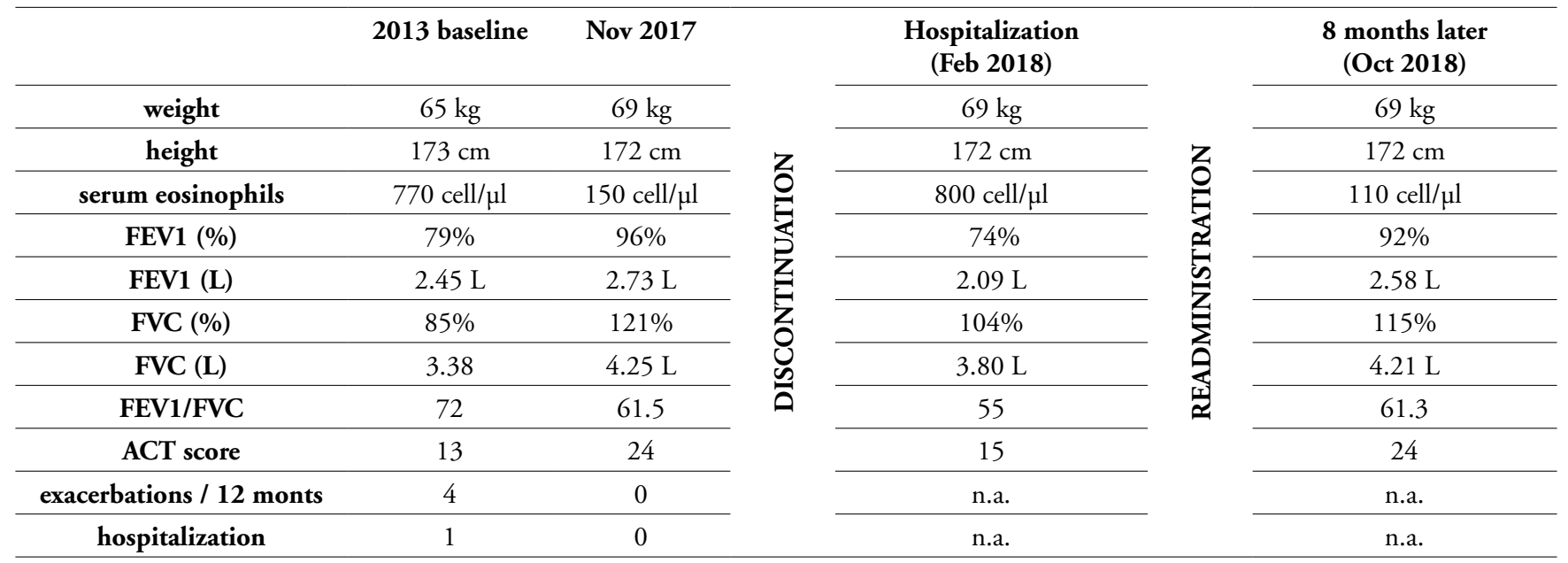

(n.a. = not available) 
The available clinical trials with MEP clearly evidenced the efficacy of the biological drug in reducing exacerbations of severe asthma. One of the most important point to be clarified is how long to continue the administration of MEP (10). In vitro observations suggested a hypothetical risk of rebound of airways eosinophilic inflammation after discontinuation (11). Also, an increased stimulus of $\mathrm{TH}_{2}$ cells to synthetize IL-5 was shown, associated to an upregulated expression of IL-5R by eosinophils and a persistence of preformed IL-5 in complex with the drug (12). In vivo, an observational study performed on 56 subject (27 on active arm) who suspend MEP after the trial, reported a slightly greater exacerbation rate at 12 months (non statistically significant) (3.1 vs. 3.9; rate ratio, 1.25; 95\% CI, 0.71-1.91; p $=0.54)$. An increase of overall frequency of severe exacerbations, only in treated patients and not in placebo, from $0.56 /$ patient at 0 to 3 months to $1.2 /$ patient $(\mathrm{p}=0.007$ ) at 3 to 6 months (rate ratio, 2.11;95\% CI, 1.76-2.54; $\mathrm{p}<0.001)$. As for exacerbations, also for blood eosinophils count, the increasing of value resulted higher in 0 to 3 months $(\mathrm{p}<0.001)$ and 3 to 6 months $(\mathrm{p}=$ $0.004)$ in treated patients (11). As previously observed in the case herein described, the increase in blood eosinophils paralleled the clinical exacerbation. To better understand also how to continue with the administration of the therapy, the experience in real life will be very useful. Indeed, consistent data are available about the patients enrolled in clinical trials with biologicals, but few data in the real-life setting, especially about the duration of the therapy. In addition, the characteristics of real-life patients resulted to be different from those described in clinical trials $(13,14)$.

The duration of the therapy is one of the main challenges in the management of these drugs. In fact, in the asthmatic patient, in which therapy is usually modulated according to the control of symptoms, the behaviour to be applied with biological drugs is not yet defined. Although it is common ground that a suspension of biologicals should be taken into account, at the moment the timing is not clear. With clinical practice it was possible to highlight that, after drug discontinuation, several patients responded by keeping control and others returning to have symptoms. A careful study of the biological parameters of patients treated with these drugs will be necessary in order to look for biomarkers that allow us to predict control of drug withdrawal. This clinical case is an interesting example of how cautiously MEP should be discontinued in clinical practice, being aware of the risk of exacerbations immediately after withdrawal.

\section{Conflict of Interest}

The authors declare that they have no conflict of interest

\section{Acknowledgements}

CIPRO (centro interprofessionale pneumologico ricerca ed organizzazione), IRCCS Policlinico San Martino, Genova, Italy.

\section{Funding}

No funding has been received for the writing or editing of this manuscript.

\section{References}

1. Menzella F, Galeone C, Lusuardi M, Simonazzi A, Castagnetti C, Ruggiero P, Facciolongo N. Near-fatal asthma responsive to mepolizumab after failure of omalizumab and bronchial thermoplasty. Ther Clin Risk Manag 2017; 13:1489-1493.

2. Chung KF, Wenzel SE, Brozek JL, Bush A, Castro M, Sterk $\mathrm{PJ}$, et al. International ERS/ATS guidelines on definition, evaluation and treatment of severe asthma. Eur Respir J 2014; 43(2):343-373.

3. Accordini S, Corsico AG, Braggion M, Gerbase MW, Gislason D, Gulsvik A, et al. The cost of persistent asthma in Europe: an international population-based study in adults. Int Arch Allergy Immunol 2013; 160(1):93-101.

4. Caminati M, Pham DL, Bagnasco D, Canonica GW. Type 2 immunity in asthma. World Allergy Organ J 2018; 11(1):13.

5. Barry LE, Sweeney J, O'Neill C, Price D, Heaney LG.The cost of systemic corticosteroid-induced morbidity in severe asthma: a health economic analysis. Respir Res 2017; 18(1):129.

6. Ortega HG, Liu MC, Pavord ID, Brusselle GG, Mark FitzGerald $\mathrm{J}$, Chetta A, et al. Mepolizumab treatment in patients with severe eosinophilic asthma. N Engl J Med 2014; 371:1198-1207.

7. Pavord ID, Korn S, Howarth P, Bleecker ER, Buhl R, Keene ON, et al. Mepolizumab for severe eosinophilic asthma (DREAM): a multicentre, double-blind, placebo-controlled trial. Lancet 2012; 380:651-659.

8. Bel EH, Wenzel SE, Thompson PJ, Prazma CM, Keene ON, Yancey SW, et al. Oral glucocorticoid-sparing effect of mepolizumab in eosinophilic asthma. N Engl J Med 2014; 371:11891197.

9. Passalacqua G, Matucci A, Vultaggio A, Bagnasco D, Mincarini $\mathrm{M}$, Maggi E, Canonica GW. The safety of monoclonal antibodies in asthma. Expert Opin Drug Saf 2016; 15(8):1087-1095.

10. Emma R, Morjaria JB, Fuochi V, Polosa R, Caruso M. Mepolizumab in the management of severe eosinophilic asthma in adults: current evidence and practical experience. Ther Adv Respir Dis 2018; 12:1753466618808490.

11. Haldar P, Brightling CE, Singapuri A, Hargadon B, Gupta S, Monteiro W, et al. Outcomes after cessation of mepolizumab therapy in severe eosinophilic asthma: a 12-month follow-up analysis. J Allergy Clin Immunol 2014; 133(3):921-923.

12. Stein ML, Villanueva JM, Buckmeier BK, Yamada Y, Filipovich AH, Assa'ad AH, Rothenberg ME. Anti-IL-5 (mepolizumab) therapy reduces eosinophil activation ex vivo and increases IL-5 and IL-5 receptor levels. J Allergy Clin Immunol 2008; 121:1473-1483.

13. Bagnasco D, Milanese M, Rolla G, Lombardi C, Bucca C, Heffler E, et al. Anti-IL-5 therapy in real life. The North-Western Italian experience 3 and comparison with the regulatory trials. World Allergy Organization Journal 2018; 11:34.

14. Caminati M, Senna G, Guerriero M, Dama AR, Chieco-Bianchi F, Stefanizzi G, et al. Omalizumab for severe allergic asthma in clinical trials and real-life studies: what we know and what we should address. Pulm Pharmacol Ther 2015; 31:28-35. 